PROCEEDINGS OF THE

AMERICAN MATHEMATICAL SOCIETY

Volume 132, Number 5, Pages 1507-1516

S 0002-9939(03)07247-2

Article electronically published on October 24, 2003

\title{
A BAILEY LATTICE
}

\author{
JEREMY LOVEJOY
}

(Communicated by Wen-Ching Winnie Li)

\begin{abstract}
We exhibit a technique for generating new Bailey pairs which leads to deformations of classical $q$-series identities, multiple series identities of the Rogers-Ramanujan type, identities involving partial theta functions, and a variety of representations for $q$-series by number-theoretic objects such as weight $3 / 2$ modular forms, ternary quadratic forms, and weighted binary quadratic forms.
\end{abstract}

\section{INTRODUCTION}

Two sequences $\left(\alpha_{n}, \beta_{n}\right)$ are said to form a Bailey pair with respect to $a$ if

$$
\beta_{n}=\sum_{r=0}^{n} \frac{\alpha_{r}}{(q ; q)_{n-r}(a q ; q)_{n+r}},
$$

where

$$
\left(b_{1}, \ldots, b_{j} ; q\right)_{n}:=\prod_{k=0}^{n-1}\left(1-b_{1} q^{k}\right) \ldots\left(1-b_{j} q^{k}\right) .
$$

The generation of such pairs has become the most effective technique in the study of basic hypergeometric series identities and their applications in combinatorics, number theory, and physics. In addition to providing proofs of the most famous theorems in the subject, the pairs and their resulting Bailey chains (see §2) naturally embed such identities in infinite families. Most notable, perhaps, are the multiple series identities of the Rogers-Ramanujan type, such as [4. Eq. (3.45)]

$$
\begin{aligned}
& \sum_{n_{k-1} \geq \ldots \geq n_{1} \geq 0} \frac{q^{n_{1}^{2}+\cdots+n_{k-1}^{2}}}{(q ; q)_{n_{k-1}-n_{k-2} \cdots(q ; q)_{n_{2}-n_{1}}(q ; q)_{n_{1}}}} \\
& =\prod_{n \neq 0, \pm k} \prod_{(\bmod 2 k+1)} \frac{1}{1-q^{n}},
\end{aligned}
$$

which generalizes the first Rogers-Ramanujan identity:

$$
\sum_{n=0}^{\infty} \frac{q^{n^{2}}}{(q ; q)_{n}}=\frac{1}{\left(q, q^{4} ; q^{5}\right)_{\infty}} .
$$

Here we specify a simple and fruitful method of generating new Bailey pairs with respect to $a q$, given a Bailey pair with respect to $a$, and present some applications.

Received by the editors January 23, 2003.

2000 Mathematics Subject Classification. Primary 33D15.

(c)2003 American Mathematical Society 
Taking walks on the resulting "Bailey lattice" leads to deformations of classical identities.

Theorem 1.1. For $k \geq 0$ we have

$$
\begin{aligned}
(q ; q)_{\infty}= & \sum_{\substack{n_{k+1} \geq \ldots \geq n_{1} \geq 0\\
}} \frac{q^{n_{k+1}\left(n_{k+1}+1\right) / 2+k n_{k+1}+n_{1}^{2}-\left(n_{2}+\ldots+n_{k+1}\right)}}{\left(-q^{n_{k+1}+1} ; q\right)_{k}} \\
& \times(-1)^{n_{1}}\left(1-q^{2 n_{1}+1}\right) \ldots\left(1-q^{2 n_{k+1}+k+1}\right) .
\end{aligned}
$$

Theorem 1.2. For $k \geq 1$ we have

$$
\begin{array}{r}
\frac{(q ; q)_{\infty}^{3}}{(-q ; q)_{k-1}}=\sum_{n_{k} \geq \ldots \geq n_{1} \geq 0} \frac{\left(1-q^{2 n_{1}+1}\right) \ldots\left(1-q^{2 n_{k}+k}\right) q^{n_{1}\left(n_{1}+1\right) / 2}}{\left(q^{2 n_{k}+1} ; q^{2}\right)_{k}} \\
\times(-1)^{n_{k}} q^{k n_{k}-\left(n_{1}+\ldots+n_{k}\right)}\left(2 n_{1}+1\right) .
\end{array}
$$

The base cases are Euler's pentagonal number theorem,

$$
\prod_{n=1}^{\infty}\left(1-q^{n}\right)=\sum_{n=-\infty}^{\infty}(-1)^{n} q^{n(3 n+1) / 2},
$$

and Jacobi's expansion,

$$
\prod_{n=1}^{\infty}\left(1-q^{n}\right)^{3}=\sum_{n=0}^{\infty}(-1)^{n}(2 n+1) q^{n(n+1) / 2} .
$$

Other infinite walks on the lattice yield identities of the Rogers-Ramanujan type, such as the following expansions for modular functions of weight 1 :

\section{Theorem 1.3.}

$$
\begin{aligned}
& \sum_{n_{k} \geq n_{k-1} \geq \ldots \geq n_{1} \geq 0} \frac{q^{n_{k}^{2}+n_{k-1}^{2}+\ldots+n_{1}^{2}}(q ; q)_{n_{1}}^{2}}{(q ; q)_{n_{k}-n_{k-1}}(q ; q)_{n_{k-1}-n_{k-2}} \ldots(q ; q)_{n_{2}-n_{1}}(q ; q)_{2 n_{1}+1}} \\
= & \frac{\left(q^{2 k+1} ; q^{2 k+1}\right)_{\infty}^{3}}{(q ; q)_{\infty}}, \\
& \sum_{n_{k} \geq n_{k-1} \geq \ldots \geq n_{1} \geq 0} \frac{q^{n_{k}\left(n_{k}+1\right) / 2+n_{k-1}\left(n_{k-1}+1\right) / 2+\ldots+n_{1}\left(n_{1}+1\right) / 2}(q ; q)_{n_{1}}}{(q ; q)_{n_{k}-n_{k-1}}(q ; q)_{n_{k-1}-n_{k-2}} \ldots(q ; q)_{n_{2}-n_{1}}\left(q ; q^{2}\right)_{n_{1}+1}} \\
= & \frac{\left(q^{k+1} ; q^{k+1}\right)_{\infty}^{3}}{(q ; q)_{\infty}\left(q ; q^{2}\right)_{\infty}} .
\end{aligned}
$$

Many results on so-called partial theta functions appeared in Ramanujan's lost notebook and are discussed at length in [3. Warnaar 16] has recently placed such identities clearly in the context of Bailey pairs, and we shall observe how taking a few steps on the lattice reveals infinite product representations for certain $q$-series with partial theta products, such as

Theorem 1.4.

$$
\begin{aligned}
& \frac{4 a}{(1+a)^{2}}+\sum_{n=1}^{\infty} \frac{(-1 ; q)_{n}^{2} q^{n}}{(a q, q / a ; q)_{n}}=\frac{4 a\left(q^{2} ; q^{2}\right)_{\infty}}{(1+a)^{2}\left(q ; q^{2}\right)_{\infty}(q, a q, q / a ; q)_{\infty}} \\
& \frac{a}{(1-a)^{2}}+\sum_{n=1}^{\infty} \frac{(q ; q)_{n-1}^{2} q^{n}}{(a q, q / a ; q)_{n}}=\frac{a(q ; q)_{\infty}^{2}}{(1-a)^{2}(a q, q / a ; q)_{\infty}}
\end{aligned}
$$


The lattice is also useful for exhibiting $q$-series whose coefficients are connected to number-theoretic objects besides infinite products. We will be led naturally to a variety of $q$-series that are weight $3 / 2$ modular forms, such as

\section{Theorem 1.5.}

$$
\begin{aligned}
\sum_{n=1}^{\infty} \frac{(-q ; q)_{n-1}(-1)^{n} q^{n(n+1) / 2}}{\left(q ; q^{2}\right)_{n}\left(1+q^{n}\right)} & =\sum_{n=0}^{\infty} n(-1)^{n} q^{n^{2}} \\
\sum_{n=0}^{\infty} \frac{(-1)^{n} q^{n(n+1) / 2}(q ; q)_{n}^{3}}{(q ; q)_{2 n+1}} & =\sum_{n=0}^{\infty}(2 n+1) q^{n^{2}+n} \\
\sum_{n=0}^{\infty} \frac{(q ; q)_{n}^{2}(q ; q)_{n+1}(-1)^{n} q^{(n+1)(n+2) / 2}}{(q ; q)_{2 n+2}} & =\sum_{n=0}^{\infty} n q^{n^{2}} .
\end{aligned}
$$

Finally, we consider connections between $q$-series and non-modular objects arising from steps on the lattice. One of the most striking examples of such a connection was noticed by Andrews, Dyson, and Hickerson [7, who showed that certain $q$-series have coefficients related to an indefinite quadratic form corresponding to the norm function in $\mathbb{Q}(\sqrt{6})$. We demonstrate how to construct series whose coefficients are determined by ternary quadratic forms and weighted binary quadratic forms, such as

\section{Theorem 1.6.}

$$
\begin{aligned}
\sum_{n=0}^{\infty} \frac{(q ; q)_{n}^{2}(-q)^{n}}{\left(q ; q^{2}\right)_{n+1}} & =\sum_{\substack{n=0 \\
r \leq n}}^{\infty} q^{n^{2}+2 n-r(r+1) / 2}(-1)^{n+r}(2 r+1) \\
\sum_{n=0}^{\infty} \frac{\left(q^{2} ; q^{2}\right)_{n}(-1)^{n} q^{2 n}}{\left(-q ; q^{2}\right)_{n}\left(1-q^{4 n+2}\right)} & =\sum_{\substack{n=0 \\
r \leq n \\
|j| \leq r}}^{\infty}(-1)^{r+j} q^{n^{2}+2 n+r^{2}+r-j^{2}}
\end{aligned}
$$

\section{The LATtice}

The following is known as Bailey's lemma, which shows how each Bailey pair engenders new Bailey pairs.

Lemma 2.1. If $\left(\alpha_{n}, \beta_{n}\right)$ form a Bailey pair with respect to $a$, then so do

$$
\alpha_{n}^{\prime}=\frac{(b, c ; q)_{n}(a q / b c)^{n} \alpha_{n}}{(a q / b, a q / c ; q)_{n}}
$$

and

$$
\beta_{n}^{\prime}=\frac{1}{(a q / b, a q / c ; q)_{n}} \sum_{j=0}^{n} \frac{(b, c ; q)_{j}(a q / b c ; q)_{n-j}(a q / b c)^{j} \beta_{j}}{(q ; q)_{n-j}} .
$$

One may then indefinitely iterate Bailey's lemma to obtain a chain of Bailey pairs, as specified below. 
Theorem 2.2 (Andrews, [4]). If $\left(\alpha_{n}, \beta_{n}\right)$ form a Bailey pair with respect to a, then

$$
\begin{aligned}
& \frac{\left(\frac{a q}{b_{k}}, \frac{a q}{c_{k}} ; q\right)_{m}}{\left(a q, \frac{a q}{b_{k} c_{k}} ; q\right)_{m}} \sum_{r \geq 0} \frac{\left(b_{1}, c_{1}, \ldots, b_{k}, c_{k}, q^{-m} ; q\right)_{r}}{\left(\frac{a q}{b_{1}}, \frac{a q}{c_{1}}, \ldots, \frac{a q}{b_{k}}, \frac{a q}{c_{k}}, a q^{m+1} ; q\right)_{r}}\left(\frac{-a^{k} q^{k+m}}{b_{1} c_{1} \ldots b_{k} c_{k}}\right)^{r} q^{-r(r-1) / 2} \alpha_{r} \\
& =\sum_{n_{k} \geq n_{k-1} \geq \ldots \geq n_{1} \geq 0} \frac{\left(q^{-m} ; q\right)_{n_{k}}\left(b_{k}, c_{k} ; q\right)_{n_{k}} \ldots\left(b_{1}, c_{1} ; q\right)_{n_{1}}}{\left(\frac{b_{k} c_{k} q^{-m}}{a}\right)_{n_{k}}\left(\frac{a q}{b_{k-1}}, \frac{a q}{c_{k-1}} ; q\right)_{n_{k}} \ldots\left(\frac{a q}{b_{1}}, \frac{a q}{c_{1}} ; q\right)_{n_{2}}} \\
& \times \frac{\left(\frac{a q}{b_{k-1} c_{k-1}} ; q\right)_{n_{k}-n_{k-1}} \ldots\left(\frac{a q}{b_{1} c_{1}} ; q\right)_{n_{2}-n_{1}}}{(q ; q)_{n_{k}-n_{k-1}} \ldots(q ; q)_{n_{2}-n_{1}}}\left(\frac{a q}{b_{k-1} c_{k-1}}\right)^{n_{k-1}} \ldots\left(\frac{a q}{b_{1} c_{1}}\right)^{n_{1}} q^{n_{k}} \beta_{n_{1}} .
\end{aligned}
$$

Several authors (1], 9], 13], 15]) have extended the Bailey chain by introducing techniques for obtaining new Bailey pairs with respect to $a / q$ from Bailey pairs with respect to $a$. Each Bailey pair then begins a Bailey lattice, which implies a doubly infinite family of identities. Here we consider a Bailey lattice that arises naturally from the inversion for Bailey pairs.

Theorem 2.3. If $\left(\alpha_{n}, \beta_{n}\right)$ is a Bailey pair with respect to a, then $\left(\alpha_{n}^{*(k)}, \beta_{n}^{*(k)}\right)$ is a Bailey pair with respect to $a q^{k}$, where

$$
\begin{aligned}
& \alpha_{n}^{*(k)}=\frac{\left(1-a q^{2 n+k}\right)\left(a q^{k} / d_{k} ; q\right)_{n}\left(-d_{k}\right)^{n} q^{n(n-1) / 2}}{\left(1-a q^{k}\right)\left(d_{k} q ; q\right)_{n}} \\
& \times \sum_{\begin{array}{l}
n \geq n_{k} \geq \ldots \geq n_{1} \geq 0 \\
\left(d_{1} ; q\right)_{n_{1}} \ldots\left(d_{k} ; q\right)_{n_{k}}
\end{array}} \frac{\left(1-a q^{2 n_{2}+1}\right) \ldots\left(1-a q^{2 n_{k}+k-1}\right)\left(a q / d_{1} ; q\right)_{n_{2} \ldots} \ldots\left(a q^{k-1} / d_{k-1} ; q\right)_{n_{k}}}{(1-a q) \ldots\left(1-a q^{k-1}\right)\left(a q / d_{1} ; q\right)_{n_{1}} \ldots\left(a q^{k} / d_{k} ; q\right)_{n_{k}}} \\
& \times \frac{\left(d_{1}-n_{1}\right.}{\left(d_{1} q ; q\right)_{n_{2}} \ldots\left(d_{k-1} q ; q\right)_{n_{k}}} d_{k-1}^{n_{k}-n_{k-1}} d_{k}^{-n_{k}}(-1)^{n_{1}} q^{-n_{1}\left(n_{1}-1\right) / 2} \alpha_{n_{1}}
\end{aligned}
$$

and

$$
\beta_{n}^{*(k)}=\frac{\left(d_{1}, \ldots, d_{k} ; q\right)_{n}}{\left(d_{1} q, \ldots, d_{k} q ; q\right)_{n}} \beta_{n} .
$$

Proof. Andrews [2] has demonstrated that the definition in (1.1) is equivalent to

$$
\alpha_{n}=\frac{\left(1-a q^{2 n}\right)(a ; q)_{n}(-1)^{n} q^{n(n-1) / 2}}{(1-a)(q ; q)_{n}} \sum_{j=0}^{n}\left(q^{-n} ; q\right)_{j}\left(a q^{n} ; q\right)_{j} q^{j} \beta_{j} .
$$

Setting $c=a q^{n+1}$ in Bailey's lemma, (2.3) implies that if $\left(\alpha_{n}, \beta_{n}\right)$ is a Bailey pair with respect to $a$, then $\left(\alpha_{n}^{*}, \beta_{n}^{*}\right)$ is a Bailey pair with respect to $a q$, where

$$
\alpha_{n}^{*}=\frac{\left(1-a q^{2 n+1}\right)(a q / b ; q)_{n}(-b)^{n} q^{n(n-1) / 2}}{(1-a q)(b q ; q)_{n}} \sum_{r=0}^{n} \frac{(b ; q)_{r}}{(a q / b ; q)_{r}}(-b)^{-r} q^{-r(r-1) / 2} \alpha_{r}
$$

and

$$
\beta_{n}^{*}=\frac{(b ; q)_{n}}{(b q ; q)_{n}} \beta_{n} .
$$

Iterating $k$ times gives the theorem.

Before continuing to applications of Theorem 2.3 it should be noted that some special cases have already been successfully employed in the study of $q$-series identities. The case $k=1, d_{1}=1$ is the so-called unit Bailey pair, which implies a significant portion of the known identities of the Rogers-Ramanujan type (see [4]). The case $k=1, d_{1}=0$ has been used implicitly in work involving mock theta 
functions $[8]$ and other studies of $q$-series related to quadratic forms ([7], [12]). The final section of [14] also contains an instance of Theorem 2.3.

\section{WALKing ON The LATtice}

3.1. The case $d_{i} \rightarrow \infty$. Observe that by inserting a Bailey pair $\left(\alpha_{n}, \beta_{n}\right)$ into Theorem 2.3 and setting all variables $d_{i} \rightarrow \infty$, we obtain a Bailey pair with respect to $a q^{k}$ in which $\beta_{n}^{*(k)}=q^{-k n} \beta_{n}$. Using this pair in the case $k=1, m \rightarrow \infty$ of Theorem 2.2 always makes the right-hand side into

$$
\sum_{n=0}^{\infty}\left(b_{1}, c_{1} ; q\right)_{n}\left(\frac{a q}{b_{1} c_{1}}\right)^{n} \beta_{n} .
$$

Therefore, such a series immediately has infinitely many representations.

Proof of Theorem [1.1. Consider (see [14]) the Bailey pair with respect to $q$,

$$
\beta_{n}=\frac{1}{\left(q^{2} ; q^{2}\right)_{n}} \quad \text { and } \quad \alpha_{n}=\frac{(-1)^{n} q^{n^{2}}\left(1-q^{2 n+1}\right)}{(1-q)} .
$$

Insert this pair into Theorem 2.3 with the $d_{i} \rightarrow \infty$ and substitute the resulting pair into Theorem 2.2 with $k=1, c_{1}=-q$, and $b_{1}, m \rightarrow \infty$. Using the identity [11 p. 236, (II.2)]

$$
\sum_{n=0}^{\infty} \frac{q^{n(n+1) / 2} z^{n}}{(q ; q)_{n}}=(-z q ; q)_{\infty}
$$

to write the resulting summation as a product completes the proof.

Proof of Theorem 1.2. Now (see [14]) take the Bailey pair with respect to 1,

$$
\alpha_{n}=\left\{\begin{array}{ll}
1 & n=0, \\
q^{n(n-1) / 2}\left(1+q^{n}\right) & n \geq 0,
\end{array} \quad \text { and } \quad \beta_{n}=\frac{(-1 ; q)_{n}}{(q ; q)_{n}\left(q ; q^{2}\right)_{n}} .\right.
$$

(The pair is misprinted in [14].) Insert this pair in Theorem 2.3 with $d_{1}=-1$ and the rest of the $d_{i} \rightarrow \infty$ and substitute the resulting pair into Theorem 2.2 with $m \rightarrow \infty, k=1, b_{1}=\sqrt{q}$, and $c_{1}=-\sqrt{q}$. Employing the $q$-Gauss summation [11. p. 236, (II.8)],

$$
\sum_{n=0}^{\infty} \frac{(a, b ; q)_{n}(c / a b)^{n}}{(c, q ; q)_{n}}=\frac{(c / a, c / b ; q)_{\infty}}{(c, c / a b ; q)_{\infty}},
$$

to write the resulting summation as a product completes the proof.

We close this section with two more examples, both related to indefinite quadratic forms. The first generalizes an expansion for a weight 1 modular form while the second generalizes a result of Andrews, Dyson, and Hickerson to which we alluded in the introduction.

Theorem 3.1. For $k \geq 1$ we have

$$
\begin{aligned}
& (q ; q)_{\infty}^{2}=\sum_{n_{k+1} \geq \ldots \geq n_{2} \geq\left|n_{1}\right| \geq 0} q^{n_{k+1}^{2}+k n_{k+1}-\left(n_{k}+\cdots+n_{3}\right)+n_{2}^{2}-n_{1}\left(3 n_{1}+1\right) / 2} \\
& \times(-1)^{n_{1}}\left(1-q^{2 n_{2}+1}\right) \cdots\left(1-q^{2 n_{k+1}+k}\right) .
\end{aligned}
$$


Proof. Take the Bailey pair with respect to 1 (see [14]),

$$
\alpha_{n}=(-1)^{n} q^{-n(n+1) / 2}\left(1+q^{n}\right) \quad \text { and } \quad \beta_{n}=\frac{(-1)^{n} q^{-n(n+1) / 2}}{(q ; q)_{n}} .
$$

Insert this pair into Theorem 2.3 with $d_{1}=0$ and the remaining $d_{i} \rightarrow \infty$, and substitute the resulting pair into Theorem 2.2 with $k=1, m, b_{1}, c_{1} \rightarrow \infty$. Using (3.1) to write the resulting summation as an infinite product completes the proof.

Theorem 3.2. For $k \geq 1$ we have

$$
\begin{aligned}
\sum_{n=0}^{\infty} \frac{q^{n(n+1) / 2}}{(-q ; q)_{n}}= & \sum_{\substack{n_{k+1} \geq \ldots \geq n_{2} \geq\left|n_{1}\right| \geq 0\\
}} \frac{q^{n_{k+1}\left(n_{k+1}-1\right) / 2+k n_{k+1}-\left(n_{k+1}+\cdots+n_{3}\right)+n_{2}^{2}-n_{1}^{2}}}{\left(q^{n_{k+1}+1} ; q\right)_{k-1}} \\
& \times(-1)^{n_{1}+n_{k+1}}\left(1-q^{2 n_{2}+1}\right) \cdots\left(1-q^{2 n_{k+1}+k}\right) .
\end{aligned}
$$

Proof. Use the Bailey pair (see [14]) with respect to 1,

$$
\alpha_{n}=\left\{\begin{array}{ll}
1 & n=0, \\
2(-1)^{n} & n>0
\end{array} \quad \text { and } \quad \beta_{n}=\frac{(-1)^{n}}{\left(q^{2} ; q^{2}\right)_{n}}\right.
$$

Insert this pair into Theorem 2.3 with $d_{1}=0$ and the remaining $d_{i} \rightarrow \infty$. Substitute the resulting pair into the $k=1, b_{1}=q, c_{1} \rightarrow \infty$, and $m \rightarrow \infty$ case of Theorem 2.2.

3.2. Bailey pairs with linear or quadratic factors. Bailey pairs with linear or quadratic factors arise naturally by inserting known pairs into the $k=1$ case of Theorem [2.3. In the sequel we consider some examples and their applications.

Theorem 3.3. The following sequences form Bailey pairs:

\begin{tabular}{|c|c|c|}
\hline$a$ & $\alpha_{n}$ & $\beta_{n}$ \\
\hline$q$ & $\frac{q^{n(n-1) / 2}\left(1-q^{2 n+1}\right)(2 n+1)}{(1-q)}$ & $\frac{(-1 ; q)_{n}^{2}}{(q ; q)_{2 n}}$ \\
\hline$q$ & $(-1)^{n} q^{n(n+1) / 2}(2 n+1)$ & $\frac{(q ; q)_{n}}{(-q ; q)_{n}\left(q^{3} ; q^{2}\right)_{n}}$ \\
\hline$q^{2}$ & $\frac{(-1)^{n} q^{n(n+1) / 2}\left(1-q^{n+1}\right)(n+1)}{(1-q)}$ & $\frac{(q ; q)_{n}}{\left(-q^{2} ; q\right)_{n}\left(q^{3} ; q^{2}\right)_{n}}$ \\
\hline$q^{2}$ & $\frac{(-1)^{n} q^{n(n+1) / 2}\left(1+q^{n+1}\right)(n+1)^{2}}{(1+q)}$ & $\frac{(q ; q)_{n}^{2}}{\left(q^{2} ; q\right)_{n}\left(q^{3} ; q^{2}\right)_{n}(-q ; q)_{n}}$ \\
\hline$q$ & $\frac{(-1)^{n} q^{n(n-1) / 2}\left(1-q^{2 n+1}\right)(n)(n+1)}{(1-q)}$ & $\frac{-2(q ; q)_{n-1}^{2}}{(q ; q)_{2 n}}$ \\
\hline
\end{tabular}

Proof. Inserting (3.2) into (2.4) and (2.5) with $b=-1$ gives the first pair. For the second pair, let $b=\sqrt{q}$, and $c=-\sqrt{q}$ in Bailey's lemma applied to the first pair. Then $\alpha_{n}{ }^{\prime}$ is clearly given by

$$
(-1)^{n} q^{n(n+1) / 2}(2 n+1)
$$

while

$$
\beta_{n}{ }^{\prime}=\frac{1}{\left(q^{3} ; q^{2}\right)_{n}} \sum_{j=0}^{n} \frac{(-1 ; q)_{n}^{2}(-q ; q)_{n-j}}{\left(q^{2} ; q^{2}\right)_{n}(q ; q)_{n-j}}(-q)^{j} .
$$


The summation is the coefficient of $z^{n}$ in

$$
\begin{aligned}
& \sum_{j=0}^{\infty} \frac{(-q ; q)_{j}}{(q ; q)_{j}} z^{j} \sum_{j=0}^{\infty} \frac{(-1 ; q)_{j}^{2}}{\left(q^{2} ; q^{2}\right)_{j}}(-z q)^{j} \\
= & \frac{(-z q ; q)_{\infty}}{(z ; q)_{\infty}} \sum_{j=0}^{\infty} \frac{(-1 ; q)_{j}^{2}}{\left(q^{2} ; q^{2}\right)_{j}}(-z q)^{j} \\
= & \frac{(-z q ; q)_{\infty}}{(z ; q)_{\infty}} \frac{(z ; q)_{\infty}}{(-z q ; q)_{\infty}} \sum_{n=0}^{\infty} \frac{(q ; q)_{n}}{(-q ; q)_{n}} z^{n}
\end{aligned}
$$

where we have applied the $q$-binomial theorem [11 p. 236, Eq. (II.3)],

$$
\sum_{n=0}^{\infty} \frac{(a ; q)_{n} z^{n}}{(q ; q)_{n}}=\frac{(a z ; q)_{\infty}}{(z ; q)_{\infty}},
$$

and the third iteration of Heine's transformation [11, p. 241, Eq. (III.3)],

$$
\sum_{n=0}^{\infty} \frac{(a, b ; q)_{n} z^{n}}{(c, q ; q)_{n}}=\frac{(a b z / c ; q)_{n}}{(z ; q)_{n}} \sum_{n=0}^{\infty} \frac{(c / a, c / b ; q)_{n}(a b z / c)^{n}}{(c, q ; q)_{n}}
$$

The remaining pairs follow from (2.4) and (2.5). For the third and fourth pairs, insert the second pair with $b=q$ and $b=-q$. For the final pair, insert the fourth pair with $b=q$ and then shift the summation in the definition of a Bailey pair to change from a pair with respect to $q^{3}$ to one with respect to $q$.

We note that the final four pairs have appeared in [6], where they were used to prove identities from Ramanujan's lost notebook.

Proof of Theorem 1.3. Letting $m \rightarrow \infty$ in the Bailey chain and substituting the second Bailey pair from Theorem 3.3 we see that the summation in the left side of the Bailey chain will be an infinite product by (1.8) whenever the specializations of the $b_{i}, c_{i}$ contribute a power of $q^{r(r+1) 2}$. Theorem 1.3 records just two of many examples of when this occurs. Specifically, let $b_{i}, c_{i} \rightarrow \infty$ for the first part, and let $b_{i} \rightarrow \infty$ and $c_{i}=-q$ for the second.

Observe that all Rogers-Ramanujan identities arising from applications of (1.8) have infinite products that are modular functions on $\Gamma_{0}(N)$, while the typical application of the triple product identity or the quintuple product identity gives functions on $\Gamma_{1}(N)$.

Proof of Theorem 1.4. Warnaar [16, Cor. 4.1] has shown that if $\left(\alpha_{n}, \beta_{n}\right)$ is a Bailey pair with respect to $q$, then

$$
\begin{aligned}
& \sum_{n=0}^{\infty} \frac{\beta_{n}(q ; q)_{2 n} q^{n}}{(a ; q)_{n+1}(q / a ; q)_{n}}-(1-q) \sum_{n=0}^{\infty} \frac{\alpha_{n}(-a)^{n} q^{-n(n-1) / 2}}{1-q^{2 n+1}} \\
= & \frac{-1}{\left(q^{2}, a, q / a ; q\right)_{\infty}} \sum_{r=1}^{\infty}(-a)^{r} q^{r(r-1) / 2} \sum_{n=0}^{\infty} \alpha_{n} q^{(1-r) n} \frac{1-q^{r(2 n+1)}}{1-q^{2 n+1}} .
\end{aligned}
$$


If we insert the first Bailey pair from Theorem 3.3 and simplify, we obtain

$$
\begin{aligned}
\sum_{n=0}^{\infty} & \frac{(-1 ; q)_{n}^{2} q^{n}}{(a q ; q)_{n}(q / a ; q)_{n}}=(1-a) \sum_{n=0}^{\infty}(2 n+1)(-a)^{n} \\
& +\frac{-1}{(q, a q, q / a ; q)_{\infty}} \sum_{r=1}^{\infty}(-a)^{r} q^{r(r-1) / 2} \sum_{n=-\infty}^{\infty}(2 n+1) q^{n(n+1) / 2-n r} \\
= & \frac{(1-a)^{2}}{(1+a)^{2}}-\frac{\sum_{r=1}^{\infty}(-a)^{r} q^{r(r-1) / 2} \sum_{n=r}^{\infty}(4 r) q^{n(n+1) / 2-n r}}{(q, a q, q / a ; q)_{\infty}} \\
= & \frac{(1-a)^{2}}{(1+a)^{2}}-\frac{\sum_{r=1}^{\infty}(-a)^{r} q^{r(r-1) / 2} \sum_{n=0}^{\infty}(4 r) q^{n(n+1) / 2-r(r-1) / 2}}{(q, a q, q / a ; q)_{\infty}} \\
= & \frac{(1-a)^{2}}{(1+a)^{2}}+\frac{4 a\left(q^{2} ; q^{2}\right)_{\infty}}{(1+a)^{2}\left(q ; q^{2}\right)_{\infty}(q, a q, q / a ; q)_{\infty}}
\end{aligned}
$$

where we have used the triple product identity [11 p. 239, (II.28)],

$$
\sum_{n=-\infty}^{\infty} z^{n} q^{n^{2}}=\left(-z q,-q / z, q^{2} ; q^{2}\right)_{\infty}
$$

to write the sum over $n$ as an infinite product. Using the final Bailey pair in Theorem 3.3 we find

$$
\begin{aligned}
\sum_{n=1}^{\infty} & \frac{(q ; q)_{n-1}^{2} q^{n}}{(a q ; q)_{n}(q / a ; q)_{n}}=-\frac{(1-a)}{2} \sum_{n=0}^{\infty}\left(n^{2}+n\right)(a)^{n} \\
& +\frac{1}{2(q, a q, q / a ; q)_{\infty}} \sum_{r=1}^{\infty}(-a)^{r} q^{r(r-1) / 2} \sum_{n=-\infty}^{\infty}\left(n^{2}+n\right)(-1)^{n} q^{n(n+1) / 2-n r} \\
= & \frac{-a}{(1-a)^{2}}+\frac{\sum_{r=1}^{\infty}(-a)^{r} q^{r(r-1) / 2} \sum_{n=r}^{\infty}\left(4 r n+2 r-4 r^{2}\right)(-1)^{n} q^{n(n+1) / 2-n r}}{2(q, a q, q / a ; q)_{\infty}} \\
= & \frac{-a}{(1-a)^{2}}+\frac{\sum_{r=1}^{\infty} r(a)^{r} q^{r(r-1) / 2} \sum_{n=0}^{\infty}(2 n+1)(-1)^{n} q^{n(n+1) / 2-r(r-1) / 2}}{(q, a q, q / a ; q)_{\infty}} \\
= & \frac{-a}{(1-a)^{2}}+\frac{a(q ; q)_{\infty}^{2}}{(1-a)^{2}(a q, q / a ; q)_{\infty}}
\end{aligned}
$$

where we have used (1.8) to write the sum over $n$ as an infinite product.

Proof of Theorem 1.5. Bailey pairs with linear or quadratic factors obviously lead to $q$-series with linear or quadratic factors. Making the right choices for the parameters in Theorem 2.2 can give theta series. Let $b_{1}=q$ and $c_{1}, m \rightarrow \infty$ in the $k=1$ case of Theorem 2.2 and insert the first three pairs from Theorem 3.3 for the equations in Theorem 1.5

3.3. Proof of Theorem 1.6. For the first identity, insert the second Bailey pair from Theorem 3.3 into (2.4) and (2.5) and let $b \rightarrow 0$ to find that $\left(\alpha_{n}, \beta_{n}\right)$ is a Bailey pair with respect to $q^{2}$, where

$$
\alpha_{n}=\frac{\left(1-q^{2 n+2}\right) q^{n^{2}+n}}{\left(1-q^{2}\right)} \sum_{r \leq n}(-1)^{r} q^{-r(r+1) / 2}(2 r+1)
$$


and

$$
\beta_{n}=\frac{(q ; q)_{n}}{(-q ; q)_{n}\left(q^{3} ; q^{2}\right)_{n}} .
$$

Using this pair in Theorem 2.2 with $k=1, b_{1}=q, m \rightarrow \infty$, and $c_{1}=-q$ gives the result. For identities involving ternary quadratic forms, we begin with Bailey pairs that resemble binary forms. For example, replacing $q$ by $q^{2}$ in the definition of a Bailey pair, it has been shown [8] that $\left(\alpha_{n}, \beta_{n}\right)$ is a Bailey pair with respect to $q^{2}$, where

$$
\alpha_{n}=\frac{q^{2 n^{2}+n}\left(1-q^{2 n+1}\right)}{(1-q)} \sum_{|j| \leq n}(-1)^{j} q^{-j^{2}} \quad \text { and } \quad \beta_{n}=\frac{1}{\left(-q^{2} ; q\right)_{2 n}} .
$$

If we insert this into (2.4) and (2.5) (remembering to replace $q$ by $q^{2}$ ) with $b=q$, we find a Bailey pair with respect to $q^{4}$,

$$
\alpha_{n}=\frac{(-1)^{n} q^{n^{2}}\left(1-q^{4 n+4}\right)}{\left(1-q^{4}\right)} \sum_{r=0}^{n} \sum_{|j| \leq r}(-1)^{r+j} q^{r^{2}+r-j^{2}}
$$

and

$$
\beta_{n}=\frac{\left(q ; q^{2}\right)_{n}}{\left(q^{3} ; q^{2}\right)_{n}\left(-q^{2} ; q\right)_{2 n}} .
$$

To complete the proof, put this pair into Theorem 2.2 with $k=1, b_{1}=q^{2}, c_{1}=-q^{2}$, and $m \rightarrow \infty$.

\section{ACKNOWLEDGEMENT}

Thanks are due to Ole Warnaar for helpful comments on earlier versions of this manuscript.

\section{REFERENCES}

[1] A. Agarwal, G. E. Andrews, and D. Bressoud, The Bailey Lattice, J. Indian Math. Soc. 51 (1987), 57-73. MR 90i:11113

[2] G. E. Andrews, Connection coefficient problems and partitions, Proc. Sympos. Pure Math. 34 (1979), 1-24. MR 80c:33004

[3] G. E. Andrews, Ramanujan's "lost" notebook I. Partial theta functions, Adv. Math. 41 (1981), 137-172. MR 83m:10034a

[4] G. E. Andrews, q-series: their development and application in analysis, number theory, combinatorics, physics, and computer algebra, CBMS Regional Conference Series in Mathematics 66. American Mathematical Society, Providence, RI, 1986. MR 88b:11063

[5] G. E. Andrews, Multiple series Rogers-Ramanujan type identities, Pacific J. Math 114 (1984), 267-283. MR 86c:11084

[6] G. E. Andrews, Bailey chains and generalized Lambert series I: Four identities of Ramanujan, Illinois J. Math. 36 (1992), 251-274. MR 93i:11020

[7] G. E. Andrews, F. J. Dyson, and D. Hickerson, Partitions and indefinite quadratic forms, Invent. Math. 91 (1988), 391-407. MR 89f:11071

[8] G. E. Andrews and D. Hickerson, Ramanujan's "Lost" notebook VII: The sixth order mock theta functions, Adv. Math. 89 (1991), 60-105. MR 92i:11027.

[9] D. M. Bressoud, The Bailey lattice: An introduction, in Ramanujan Revisited, pp. 57-67, Academic Press, Boston, MA, 1988. MR 89f:05018

[10] F. Franklin, Sur le développement du produit infini $(1-x)\left(1-x^{2}\right)\left(1-x^{3}\right) \ldots$, Comptes Rendus 82 (1881), 448-450.

[11] G. Gasper and M. Rahman, Basic Hypergeometric Series, Cambridge University Press, Cambridge, 1990. MR 91d:33034

[12] J. Lovejoy, Lacunary partition functions, Math. Res. Lett. 9 (2002), 191-198. MR 2003f:11157 
[13] A. Schilling and S. O. Warnaar, A higher level Bailey lemma: proof and application, Ramanujan J. 2 (1998), 327-349. MR 99k:11028

[14] L. J. Slater, A new proof of Rogers's transformations of infinite series, Proc. London Math. Soc. (2) 53 (1951), 460-475. MR 13:227g

[15] S. O. Warnaar, 50 years of Bailey's lemma, Algebraic Combinatorics and Applications, Springer-Verlag, Berlin, 2001, pp. 333-347. MR 2002g:33020

[16] S. O. Warnaar, Partial theta functions I: beyond the lost notebook, preprint.

CNRS, LaBRi, Université Bordeaux I, 351 Cours de la libération, 33405 Talence Cedex, France

E-mail address: lovejoy@labri.fr 